\title{
Plasmonic Slot Nano-Resonators in Gold-Coated Microfibers
}

\author{
Ming Ding*, Gilberto Brambilla, Michalis N. Zervas \\ Optoelectronics Research Centre, University of Southampton, Southampton SO17 1BJ, United Kingdom
}

In recent years, research in plasmonics and related devices has attracted considerable interest. Nanoscale apertures in thin noble-metal films, with dimensions comparable to the light wavelength, can form plasmonic nano-resonators (PNRs) and such structures can concentrate an incident light field into a small volume which overcomes the diffraction limit with orders-of-magnitude intensity enhancement. So far, the transmission properties of plasmonic slot nano-resonators (PSNRs) have been studied under plane-wave excitation directed perpendicularly to the plane of the resonator $[1,2]$.

In this paper, we theoretically study a strongly-coupled 3D PSNR by embedding a slot nano-cavity in a plasmonic cylindrical waveguide formed by a thin-gold-film coated microfiber using 3D Finite Element Method. Light is launched from one end of the microfiber with $1 \mu \mathrm{m}$ diameter and $30 \mathrm{~nm}$ gold layer and the various resonances can be identified simply monitoring the transmitted and reflected light. Fig. 1 (a) shows the top-view of the bow-tie nano-cavity and Fig. 1 (b) shows the transmissivity and the reflectivity of the composite structure with the bow-tie PSNR geometry with $L=400 \mathrm{~nm}, D=200 \mathrm{~nm}$, and $d=34.4 \mathrm{~nm}$. Three main resonances were identified: the resonance at $\lambda=880 \mathrm{~nm}$ corresponds to a second-order resonance, with two intensity maxima along the length of the bow-tie PSNR. The resonances at $\lambda=1524 \mathrm{~nm}$ and $2050 \mathrm{~nm}$ correspond to first-order PSNR resonances, with only one intensity maximum along the bow-tie length. Fig. 1 (c) shows the total electric field modulus at several wavelengths corresponding to the major ( $\lambda=880 \mathrm{~nm}, 1524 \mathrm{~nm}$ and $2050 \mathrm{~nm})$ resonances, at which the electric field is highly localized and centered at the bow-tie waist. Shown are also some minor $(\lambda=820$ $\mathrm{nm}, 970 \mathrm{~nm}, 1040 \mathrm{~nm}, 1170 \mathrm{~nm}$ and $1260 \mathrm{~nm}$ ) resonances, whose corresponding electric field distributions are gradually shifting off-center and their peak values decrease.

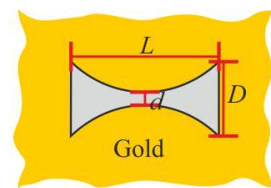

(a)

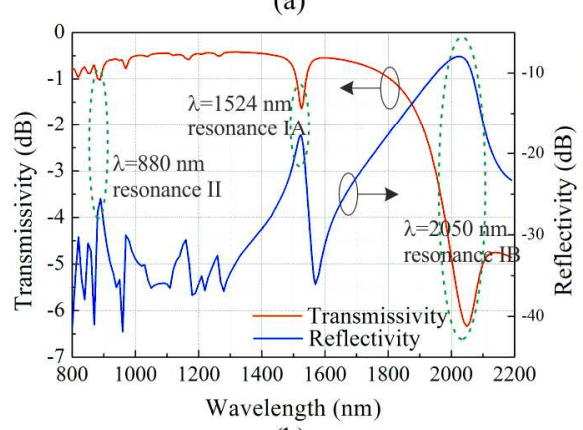

(b)

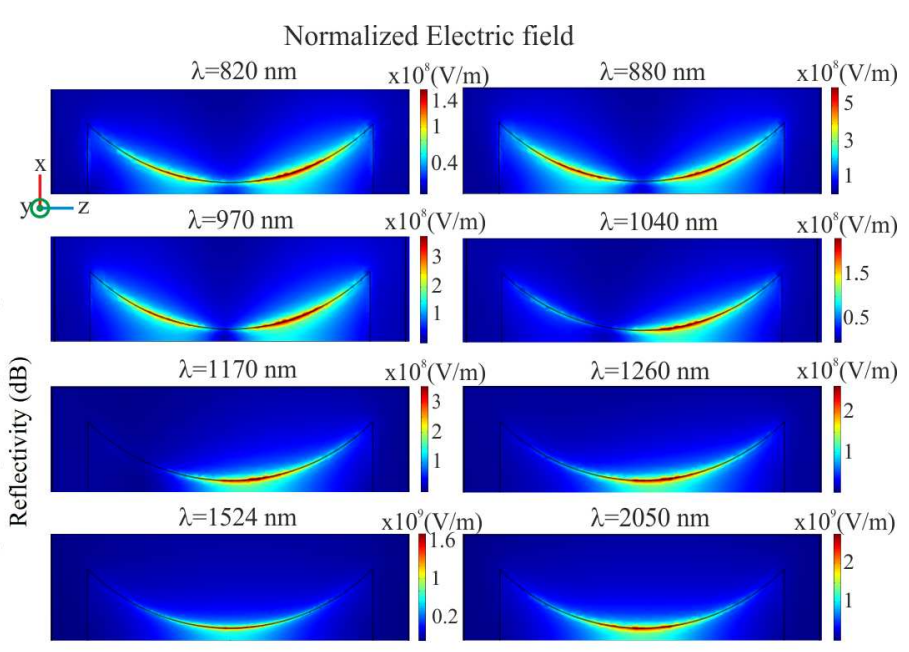

(c)

Fig. 1 (a) Schematic of bow-tie nano-cavity; (b) Transmissivity (red) and reflectivity (blue) of the bow-tie PSNR embedded in the plasmonics microfiber; (c) The normalized electric field on X-Z plane for bow-tie SNR at different wavelengths $\lambda$.

Different bow-tie PSNRs with different waist and different edge width, multiple cascaded bow-tie PSNR, and rectangular PSNR were numerically investigated. A single resonator shows enhancement factor in excess of $9 \times 10^{5}$, which we believe is the biggest enhancement factor calculated in all types of nano-resonators so far. Wavelength shift rates were found to be strongly dependent on the nature of the associated resonance and the plasmonic waveguide characteristics. It was observed that resonances lying closer to the plasmonic waveguide fundamental mode cut-off are much more sensitive on the PSNR dimensions. In addition to higher wavelengthshift sensitivities, the longer-wavelength resonances showed also the largest intensity enhancement factors. Using multiple cascaded PSNR structures results in even higher intensity enhancement factors (in excess of one million) in multiple spots. The combination of these features implies that designs that are based on longerwavelength resonances are the most promising candidates for advanced applications such as SERS, optical filtering, spectroscopy and bio-sensing.

\section{References}

[1] S. Sederberg and A. Y. Elezzabi, "Nanoscale plasmonic contour bowtie antenna operating in the mid-infrared," Opt Express 19, 1553215537 (2011).

[2] E. X. Jin and X. F. Xu, "Enhanced optical near field from a bowtie aperture," Appl. Phys. Lett. 88, 153110 (2006). 